\title{
Número necessário de experimentos para a análise de trilha em feijão
}

\author{
Number of necessary experiments for the path analysis in common bean
}

\author{
Alberto Cargnelutti Filho ${ }^{\mathrm{I} *}$ Nerinéia Dalfollo Ribeiro ${ }^{\mathrm{I}}$ Cláudia Burin $^{\mathrm{II}}$ Marcos Toebe $^{\mathrm{III}}$ \\ Gabriele Casarotto ${ }^{\mathrm{IV}}$
}

\section{RESUMO}

A fim de obter confiabilidade nas estimativas, geradas por meio da análise de trilha, é importante definir o número necessário de experimentos. $O$ objetivo deste trabalho foi determinar o número de experimentos necessários para a análise de trilha do número de vagens por planta, do número de sementes por vagem e da massa de cem grãos sobre a produtividade de grãos de cultivares de feijão (Phaseolus vulgaris $L$.). Treze cultivares de feijão foram avaliadas em nove experimentos conduzidos em Santa Maria, Estado do Rio Grande do Sul, entre os anos agrícolas de 2000/2001 e 2004/ 2005. Foram mensurados os caracteres produtividade de grãos, número de vagens por planta, número de sementes por vagem e massa de cem grãos. Foram planejadas 511 matrizes de dados (13 cultivares e 4 caracteres) e realizadas análises de correlação, de diagnóstico de multicolinearidade e de trilha, além de construir diagramas de dispersão. $O$ número de experimentos, necessários para a análise de trilha, foi determinado a partir das estimativas dos parâmetros do modelo quadrático de resposta com platô. Na região da depressão central do Estado do Rio Grande do Sul, sete experimentos são suficientes para a análise de trilha em feijão.

Palavras-chave: Phaseolus vulgaris L., análise de correlação, modelo quadrático de resposta com platô.

\section{ABSTRACT}

In order to obtain reliable estimates, generated through path analysis, it is important to define the necessary number of experiments. The objective of this work was to determine the number of experiments necessary for the path analysis the number of pods per plant, number seed per pod and weight of 100 grains on grain yield the common bean cultivars (Phaseolus vulgaris L.). Thirteen common bean cultivars were evaluated in nine experiments conducted in Santa Maria, in Rio Grande do Sul State, Brazil, in the growing seasons between 2000/2001 and 2004/2005. We measured the characters grain yield, number of pods per plant, number seed per pod and weight of 100 grains. Were planned 511 data matrices (13 cultivars and four characters). Analyses of correlation, diagnoses of multicollinearity, path analysis and scatter plots were carried out. The number of experiments necessary for the path analysis, was determined by the estimates of the parameters of the quadratic response plateau model. In the central depression region of Rio Grande do Sul State, Brazil, seven experiments are sufficient for the path analysis in common bean.

Key words: Phaseolus vulgaris L., correlation analysis, quadratic response plateau model.

\section{INTRODUÇÃO}

As relações lineares entre um conjunto de caracteres podem ser investigadas por meio do coeficiente de correlação linear de Pearson e por outros procedimentos estatísticos complementares, como, por exemplo, a análise de trilha. A análise de trilha permite desdobrar esses coeficientes em efeitos diretos e indiretos de variáveis explicativas sobre uma variável principal (CRUZ \& REGAZZI, 1997; CRUZ \& CARNEIRO, 2003).

'Departamento de Fitotecnia, Centro de Ciências Rurais (CCR), Universidade Federal de Santa Maria (UFSM), 97105-900, Santa Maria, RS, Brasil. E-mail: cargnelutti@pq.cnpq.br. *Autor para correspondência.

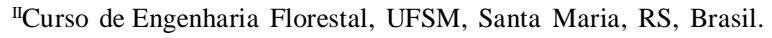

IIIPrograma de Pós-graduação em Agronomia, UFSM, Santa Maria, RS, Brasil.

${ }^{\mathrm{Iv}}$ Curso de Agronomia, UFSM, Santa Maria, RS, Brasil. 
Análises de trilha, comumente realizadas a partir de dados de um experimento, têm sido utilizadas na cultura de feijoeiro (DUARTE \& ADAMS, 1972; COIMBRA et al., 1999; KUREK et al., 2001; FURTADO et al., 2002; DURSUN, 2007). De maneira geral, nesses trabalhos, a análise de trilha tem destacado que há associação linear de causa e efeito entre o caráter número de vagens por planta e a produtividade de grãos, evidenciando que o número de vagens por planta é um caráter adequado para a seleção indireta. A interação cultivares $\times$ ambientes é esperada em experimentos conduzidos em diferentes locais, anos e épocas de cultivo. Então, os resultados da análise de trilha podem ser alterados com a inclusão de novos ambientes (locais, anos e épocas). Assim, a análise de trilha, com base em apenas um experimento, poderá ser pouco eficiente devido à variabilidade de resposta das cultivares frente às condições ambientais.

O número necessário de experimentos para a análise de agrupamento (CARGNELUTTI FILHO et al., 2009a) e para a comparação de cultivares de feijão (CARGNELUTTI FILHO et al., 2006) tem sido investigado. No entanto, o dimensionamento do número de experimentos para a análise de trilha em feijão não foi encontrado na literatura.

O objetivo deste trabalho foi determinar o número de experimentos necessários para a análise de trilha do número de vagens por planta, do número de sementes por vagem e da massa de cem grãos sobre a produtividade de grãos de cultivares de feijão (Phaseolus vulgaris L.), na região da depressão central do Estado do Rio Grande do Sul.

\section{MATERIAL E MÉTODOS}

Foram avaliadas 13 cultivares de feijão 'Carioca', 'Diamante Negro', 'TPS Nobre', 'Guapo Brilhante', 'Guateian 6662', 'IAPAR 44', 'Macanudo', 'Macotaço', 'Minuano', 'Pérola', 'Rio Tibagi', 'TPS Bionobre' e 'TPS Bonito' - em nove experimentos, realizados em delineamento de blocos ao acaso, com três repetições, na Universidade Federal de Santa Maria, Santa Maria, Rio Grande do Sul (29\%42S, 5349W, a $95 \mathrm{~m}$ de altitude). As parcelas foram compostas de quatro fileiras de $4 \mathrm{~m}$ de comprimento, espaçadas de $0,50 \mathrm{~m}$, e a área útil de $3 \mathrm{~m}^{2}$. Os experimentos foram conduzidos nos anos agrícolas 2000/2001 a 2004/2005, em duas épocas de cultivo: safra (semeadura em setembro ou outubro) e safrinha (semeadura em janeiro ou fevereiro). Em cada experimento, foram mensurados os caracteres produtividade de grãos a 13\% de umidade (PROD), número de vagens por planta (NVP), número de sementes por vagem (NSV) e massa de cem grãos (MCG).
Inicialmente, foi feito um diagrama de dispersão das 351 observações (13 cultivares/ experimento $\mathrm{x}$ três observações (repetições)/cultivar $\mathrm{x}$ nove experimentos) dos caracteres produtividade de grãos, número de vagens por planta, número de sementes por vagem e massa de cem grãos, a fim de investigar, visualmente, a relação entre eles. Após, foram organizados 511 arquivos de dados, com as médias dos caracteres PROD, NVP, NSV e MCG de cada uma das cultivares, sendo nove formados pelos dados individuais de cada experimento, e os demais pela combinação dos nove experimentos em grupos de dois (36), três (84), quatro (126), cinco (126), seis (84), sete (36), oito (9) e nove (1 arquivo) experimentos. A seguir, em cada um desses 511 arquivos de dados, foi determinada a matriz de coeficientes de correlação linear de Pearson (matriz fenotípica) entre os caracteres PROD, NVP, NSV e MCG, e verificada a significância dos coeficientes por meio do teste $\mathrm{t}$ de Student, a $5 \%$ de probabilidade, com 11 graus de liberdade. Em seguida, foi realizado o diagnóstico de multicolinearidade (CRUZ, 2006), conforme critério de MONTGOMERY \& PECK (1982), e a análise de trilha (path analysis) da variável principal ou endógena PROD em função das variáveis explicativas ou exógenas NVP, NSV e MCG, conforme metodologia descrita em CRUZ \& REGAZZI (1997).

As estimativas dos coeficientes de correlação linear de Pearson dos pares de caracteres (NVP e PROD, NSV e PROD e MCG e PROD), entre os nove experimentos, foram comparadas por meio do teste $\chi^{2}$, a $5 \%$ de probabilidade, conforme metodologia descrita em FERREIRA (2009). A fim de avaliar, visualmente, a variabilidade das estimativas dos coeficientes de correlação linear de Pearson e respectivos efeitos diretos e indiretos dos caracteres número de vagens por planta, número de sementes por vagem e massa de cem grãos sobre a produtividade de grãos, bem como do coeficiente de determinação, da variável residual e do número de condição, em função do número de experimentos, foram feitos diagramas de dispersão.

Para investigar o número necessário de experimentos para a análise de trilha, foram obtidas as estimativas dos parâmetros do modelo quadrático de resposta com platô das variáveis Y (coeficientes de correlação linear de Pearson entre NVPe PROD, NSV e PROD e MCG e PROD) em função da variável X (número de experimentos). Nesse modelo, o ponto de início do platô é denominado de $\mathrm{X}_{0}$ (número necessário de experimentos), que é localizado por meio da primeira derivada da equação quadrática, isto é, pelo ponto de máximo ou de mínimo. Quando X é maior ou igual a $\mathrm{X}_{0}$, 
este ponto se torna uma constante, transformando-se numa reta com inclinação zero, denominada platô, cujo modelo é dado por $\mathrm{Y}=\mathrm{p}$, em que $\mathrm{p}=$ platô; e quando $\mathrm{X}$ é menor que $\mathrm{X}_{0}$, este ponto se encontra na parte quadrática do modelo $\left(\mathrm{Y}=\mathrm{a}+\mathrm{bX}+\mathrm{cX} \mathrm{X}^{2}\right)$, sendo descrito por uma parábola (REZENDE et al., 2007). Como as demais estatísticas, relacionadas à análise de trilha, dependem dos coeficientes de correlação linear de Pearson, considerou-se a média dos três valores de $\mathrm{X}_{0}$ como sendo o número necessário de experimentos para a análise de trilha.

\section{RESULTADOS E DISCUSSÃO}

O diagrama de dispersão entre os caracteres produtividade de grãos (PROD), número de vagens por planta (NVP), número de sementes por vagem (NSV) e massa de cem grãos (MCG) sugere, de maneira geral, que há relações de linearidade entre eles, principalmente relações lineares positivas entre PROD versus NVP, NSV e MCG (Figura 1). Portanto, embora a relação entre as variáveis explicativas (NVP, NSV e MCG) e a variável principal (PROD) seja estruturalmente multiplicativa
(CRUZ \& REGAZZI, 1997), o estudo de relações lineares entre esses caracteres, como, por exemplo, por meio de análises de correlação e de trilha, sem a transformação dos dados para a escala logarítmica, é adequado (HAIR et al., 2005).

O diagnóstico de multicolinearidade, nas 511 matrizes de coeficientes de correlação linear de Pearson entre os caracteres número de vagens por planta, número de sementes por vagem e massa de cem grãos, revelou que o número de condição (NC) oscilou entre 5 e 70 (Tabelas 1 e 2 e Figura 2), o que não constitui problema para a análise de trilha, conforme critério de MONTGOMERY \& PECK (1982). Portanto, o efeito adverso da multicolinearidade está contornado, o que permite a realização adequada da análise de trilha (CRUZ \& CARNEIRO, 2003; CRUZ, 2006). Assim, foram realizadas 511 análises de trilha da variável principal PROD em função das variáveis explicativas NVP, NSV e MCG (Tabelas 1 e 2 e Figuras 2 e 3).

Entre os nove experimentos, a estimativa do coeficiente de correlação linear de Pearson (r) entre NVPe PROD, oscilou de 0,0847 (experimento 7 - Safra 2003/2004) a 0,8279 (experimento 4 - Safrinha 2002). Entre

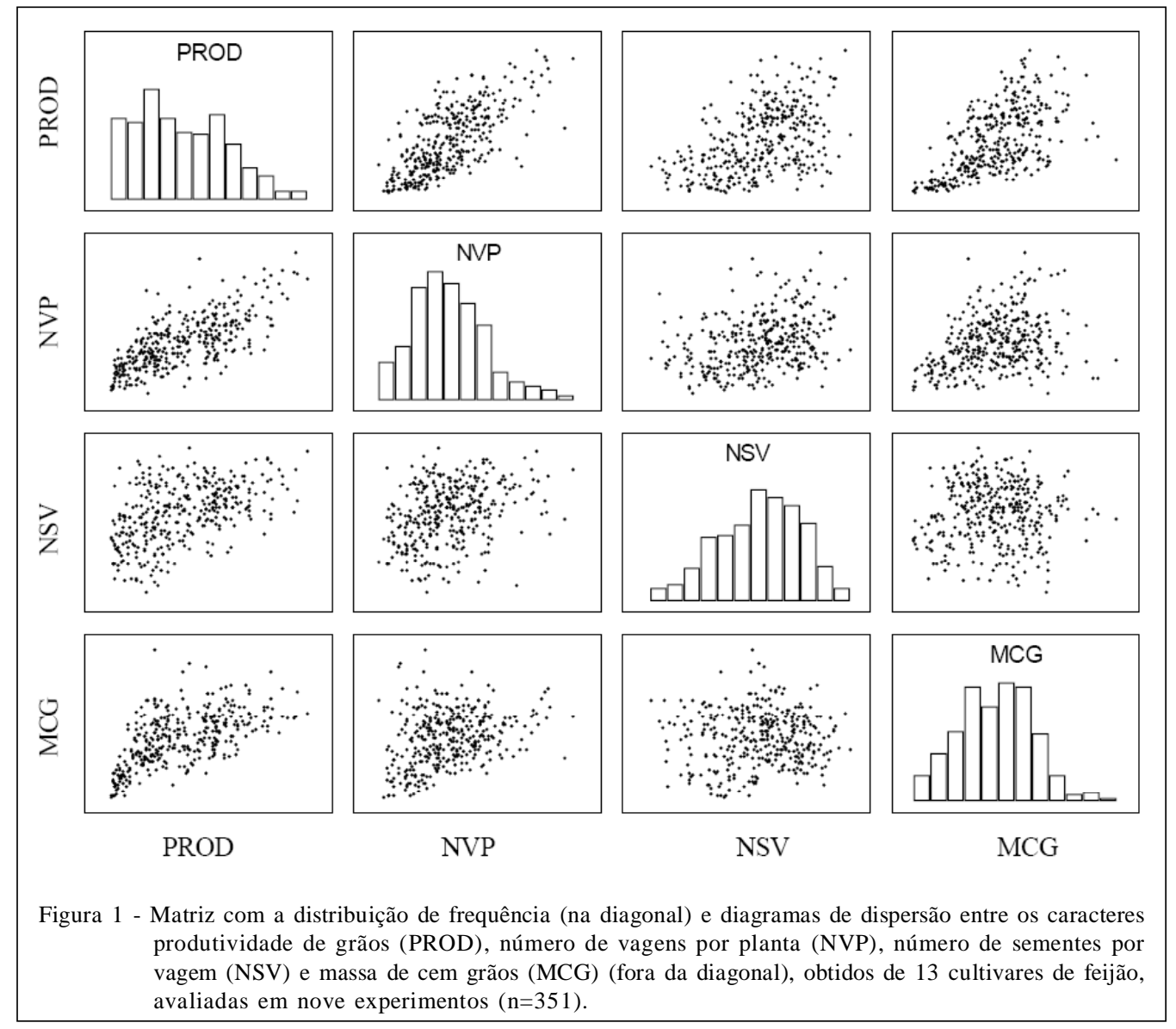

Ciência Rural, v.41, n.4, abr, 2011. 
Tabela 1 - Estimativas dos coeficientes de correlação de Pearson e respectivas estimativas dos efeitos diretos e indiretos dos caracteres número de vagens por planta (NVP), número de sementes por vagem (NSV) e massa de cem grãos (MCG) sobre a produtividade de grãos (PROD) de 13 cultivares de feijão, avaliadas em nove experimentos.

\begin{tabular}{|c|c|c|c|c|c|c|}
\hline \multirow{2}{*}{$\begin{array}{l}\text { Variável } \\
\text { explicativa }\end{array}$} & \multirow{2}{*}{ Efeito } & \multicolumn{5}{|c|}{ - Experimento ${ }^{(1)}$} \\
\hline & & 1 & 2 & 3 & 4 & 5 \\
\hline \multirow[t]{4}{*}{ NVP } & Direto sobre PROD & 0,9539 & 0,5431 & 0,5714 & 0,6547 & 0,5641 \\
\hline & Indireto via NSV & 0,0009 & 0,0640 & $-0,0755$ & $-0,0002$ & 0,2284 \\
\hline & Indireto via MCG & $-0,4306$ & 0,0291 & 0,0267 & 0,1734 & 0,0159 \\
\hline & Total (correlação Pearson) ${ }^{(2)}$ & $0,5242^{\text {ns }} a b c$ & $0,6362 * a b c$ & $0,5226^{\text {ns }}$ abc & $0,8279^{*} \mathrm{a}$ & $0,8084 * a b$ \\
\hline \multirow[t]{4}{*}{ NSV } & Direto sobre PROD & 0,0017 & 0,1650 & 0,1838 & 0,0597 & 0,3456 \\
\hline & Indireto via NVP & 0,5253 & 0,2106 & $-0,2347$ & $-0,0021$ & 0,3728 \\
\hline & Indireto via MCG & $-0,1700$ & $-0,0831$ & $-0,0578$ & $-0,1830$ & $-0,0670$ \\
\hline & Total (correlação Pearson) ${ }^{(2)}$ & $0,3570^{\mathrm{ns}} \mathrm{ab}$ & $0,2925^{\mathrm{ns}} \mathrm{ab}$ & $-0,1087^{\text {ns }} b c$ & $-0,1254^{\text {ns }} b c$ & $0,6514 * a$ \\
\hline \multirow[t]{9}{*}{ MCG } & Direto sobre PROD & 0,6921 & 0,2512 & 0,1369 & 0,3987 & 0,3320 \\
\hline & Indireto via NVP & $-0,5935$ & 0,0629 & 0,1115 & 0,2848 & 0,0270 \\
\hline & Indireto via NSV & $-0,0004$ & $-0,0546$ & $-0,0776$ & $-0,0274$ & $-0,0698$ \\
\hline & Total (correlação Pearson) ${ }^{(2)}$ & $0,0982^{\text {ns }} b$ & $0,2596^{\mathrm{ns}} \mathrm{ab}$ & $0,1708^{\mathrm{ns}} \mathrm{ab}$ & $0,6561^{*} \mathrm{a}$ & $0,2893^{\mathrm{ns}} \mathrm{ab}$ \\
\hline & Coeficiente de determinação & 0,5687 & 0,4590 & 0,3020 & 0,7961 & 0,7771 \\
\hline & Variável residual & 0,6568 & 0,7355 & 0,8354 & 0,4515 & 0,4721 \\
\hline & Número de condição & 17 & 5 & 5 & 19 & 18 \\
\hline & & \multicolumn{4}{|c|}{-'-1- } & Geral \\
\hline & & 6 & 7 & 8 & 9 & \\
\hline \multirow[t]{4}{*}{ NVP } & Direto sobre PROD & 0,3526 & 0,2132 & 0,0097 & 0,4079 & 0,8373 \\
\hline & Indireto via NSV & 0,0676 & $-0,1006$ & $-0,0900$ & 0,0343 & $-0,0318$ \\
\hline & Indireto via MCG & 0,1265 & $-0,0279$ & 0,2294 & $-0,0247$ & $-0,0066$ \\
\hline & Total (correlação Pearson) ${ }^{(2)}$ & $0,5467^{\text {ns }} a b c$ & $0,0847^{\text {ns }} c$ & $0,1492^{\text {ns }} c$ & $0,4175^{\mathrm{ns}} \mathrm{bc}$ & $0,7989 *$ \\
\hline \multirow[t]{4}{*}{ NSV } & Direto sobre PROD & 0,2828 & 0,3790 & 0,2482 & $-0,2723$ & 0,3316 \\
\hline & Indireto via NVP & 0,0843 & $-0,0566$ & $-0,0035$ & $-0,0514$ & $-0,0804$ \\
\hline & Indireto via $\mathrm{MCG}$ & 0,0782 & $-0,4097$ & $-0,2566$ & $-0,1002$ & $-0,3674$ \\
\hline & Total (correlação Pearson) ${ }^{(2)}$ & $0,4453^{\text {ns }} a b$ & $-0,0873^{\text {ns }} b c$ & $-0,0119^{\text {ns }} b c$ & $-0,4238^{\text {ns }} c$ & $-0,1162^{\mathrm{ns}}$ \\
\hline \multirow[t]{7}{*}{ MCG } & Direto sobre PROD & 0,3697 & 0,8519 & 0,7456 & 0,5553 & 0,6011 \\
\hline & Indireto via NVP & 0,1207 & $-0,0070$ & 0,0030 & $-0,0182$ & $-0,0091$ \\
\hline & Indireto via NSV & 0,0598 & $-0,1823$ & $-0,0854$ & 0,0491 & $-0,2026$ \\
\hline & Total (correlação Pearson) ${ }^{(2)}$ & $0,5502^{\mathrm{ns}} \mathrm{ab}$ & $0,6626^{*} \mathrm{a}$ & $0,6632 * \mathrm{a}$ & $0,5863^{*} \mathrm{ab}$ & $0,3894^{\mathrm{ns}}$ \\
\hline & Coeficiente de determinação & 0,5221 & 0,5494 & 0,4930 & 0,6113 & 0,8644 \\
\hline & Variável residual & 0,6913 & 0,6712 & 0,7120 & 0,6235 & 0,3682 \\
\hline & Número de condição & 7 & 10 & 8 & 8 & 35 \\
\hline
\end{tabular}

(1) 1, Safra 2000/2001; 2, Safrinha 2001; 3, Safra 2001/2002; 4, Safrinha 2002; 5, Safra 2002/2003; 6, Safrinha 2003; 7, Safra 2003/2004; 8,

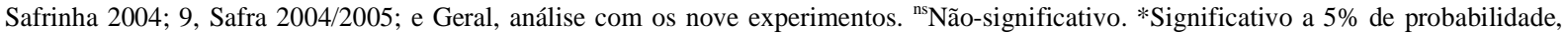
pelo teste t, com 11 graus de liberdade. ${ }^{(2)}$ Coeficiente de correlação linear de Pearson entre NVP e PROD, NSV e PROD e MCG e PROD, não seguidos de mesma letra, na linha (comparação entre experimentos), diferem a 5\% de probabilidade, pelo teste $\chi^{2}$.

NSV e PROD, or variou de -0,4238 (experimento 9 Safra 2004/2005) a 0,6514 (experimento 5 - Safra 2002/ 2003). Já entre MCG e PROD o intervalo do $r$ foi de 0,0982 (experimento 1 - Safra 2000/2001) a 0,6632 (experimento 8 - Safrinha 2004) (Tabela 1). A magnitude dessas amplitudes de variação entre os nove experimentos, para cada par de caracteres, sugere diferenças entre os experimentos em relação ao coeficiente de correlação. $O$ teste de $\chi^{2}$ (FERREIRA, 2009) aplicado nas nove estimativas de r, de cada par 
Tabela 2 - Estimativas dos coeficientes de correlação (r) e respectivas estimativas dos efeitos diretos (Dir) e indiretos (Ind) dos caracteres explicativos (CE) número de vagens por planta (NVP), número de sementes por vagem (NSV) e massa de cem grãos (MCG) sobre a produtividade de grãos (PROD) de 13 cultivares de feijão, em 36 combinações de nove experimentos em grupos de sete experimentos.

\begin{tabular}{|c|c|c|c|c|c|c|c|c|c|c|c|c|c|}
\hline \multirow{2}{*}{$\mathrm{CE}$} & \multirow{2}{*}{ Efeito } & \multicolumn{12}{|c|}{ 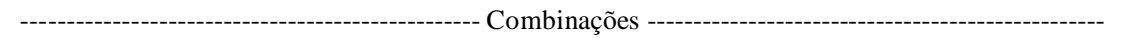 } \\
\hline & & 1 & 2 & 3 & 4 & 5 & 6 & 7 & 8 & 9 & 10 & 11 & 12 \\
\hline \multirow[t]{4}{*}{ NVP } & Dir sobre PROD & 0,80 & 0,80 & 0,83 & 0,81 & 0,84 & 0,83 & 0,78 & 0,86 & 0,82 & 0,86 & 0,81 & 0,85 \\
\hline & Ind via NSV & 0,02 & 0,03 & 0,00 & 0,03 & $-0,03$ & 0,01 & 0,00 & $-0,07$ & $-0,02$ & $-0,06$ & 0,03 & $-0,03$ \\
\hline & Ind via MCG & $-0,01$ & 0,00 & 0,01 & $-0,02$ & $-0,01$ & 0,01 & $-0,03$ & $-0,03$ & 0,00 & $-0,03$ & $-0,07$ & $-0,06$ \\
\hline & $\mathrm{r}$ & 0,81 & 0,84 & 0,84 & 0,82 & 0,80 & 0,84 & 0,75 & 0,76 & 0,80 & 0,77 & 0,76 & 0,76 \\
\hline \multirow[t]{4}{*}{ NSV } & Dir sobre PROD & 0,34 & 0,20 & 0,24 & 0,33 & 0,36 & 0,23 & 0,32 & 0,38 & 0,22 & 0,36 & 0,24 & 0,25 \\
\hline & Ind via NVP & 0,05 & 0,13 & $-0,02$ & 0,07 & $-0,06$ & 0,02 & 0,00 & $-0,16$ & $-0,09$ & $-0,14$ & 0,09 & $-0,10$ \\
\hline & Ind via MCG & $-0,36$ & $-0,26$ & $-0,26$ & $-0,40$ & $-0,41$ & $-0,29$ & $-0,38$ & $-0,38$ & $-0,28$ & $-0,43$ & $-0,32$ & $-0,29$ \\
\hline & $\mathrm{r}$ & 0,04 & 0,07 & $-0,04$ & 0,00 & $-0,11$ & $-0,04$ & $-0,06$ & $-0,16$ & $-0,15$ & $-0,21$ & 0,01 & $-0,14$ \\
\hline \multirow[t]{8}{*}{ MCG } & Dir sobre PROD & 0,57 & 0,45 & 0,47 & 0,59 & 0,61 & 0,48 & 0,63 & 0,66 & 0,53 & 0,68 & 0,54 & 0,53 \\
\hline & Ind via NVP & $-0,02$ & 0,01 & 0,02 & $-0,03$ & $-0,02$ & 0,01 & $-0,04$ & $-0,04$ & 0,00 & $-0,04$ & $-0,11$ & $-0,10$ \\
\hline & Ind via NSV & $-0,21$ & $-0,11$ & $-0,14$ & $-0,22$ & $-0,24$ & $-0,14$ & $-0,19$ & $-0,22$ & $-0,11$ & $-0,23$ & $-0,14$ & $-0,14$ \\
\hline & $\mathrm{r}$ & 0,34 & 0,34 & 0,35 & 0,34 & 0,35 & 0,35 & 0,40 & 0,40 & 0,41 & 0,41 & 0,28 & 0,29 \\
\hline & Coef. determinação & 0,85 & 0,84 & 0,86 & 0,86 & 0,84 & 0,85 & 0,82 & 0,86 & 0,84 & 0,87 & 0,77 & 0,77 \\
\hline & Variável residual & 0,39 & 0,40 & 0,38 & 0,38 & 0,39 & 0,38 & 0,42 & 0,38 & 0,40 & 0,37 & 0,48 & 0,48 \\
\hline & Número de condição & 28 & 23 & 28 & 30 & 31 & 28 & 24 & 36 & 27 & 39 & 17 & 19 \\
\hline & & 13 & 14 & 15 & 16 & 17 & 18 & 19 & 20 & 21 & 22 & 23 & 24 \\
\hline \multirow[t]{4}{*}{ NVP } & Dir sobre PROD & 0,82 & 0,83 & 0,85 & 0,79 & 0,80 & 0,80 & 0,81 & 0,79 & 0,81 & 0,82 & 0,90 & 0,84 \\
\hline & Ind via NSV & 0,00 & $-0,01$ & $-0,04$ & 0,05 & 0,02 & 0,03 & 0,03 & 0,01 & 0,02 & $-0,07$ & $-0,16$ & $-0,07$ \\
\hline & Ind via MCG & $-0,04$ & $-0,06$ & $-0,09$ & $-0,02$ & $-0,01$ & 0,01 & $-0,03$ & $-0,03$ & $-0,08$ & 0,00 & 0,00 & 0,02 \\
\hline & $\mathrm{r}$ & 0,79 & 0,76 & 0,71 & 0,83 & 0,81 & 0,83 & 0,81 & 0,77 & 0,76 & 0,74 & 0,73 & 0,79 \\
\hline \multirow[t]{4}{*}{ NSV } & Dir sobre PROD & 0,11 & 0,20 & 0,24 & 0,18 & 0,27 & 0,12 & 0,25 & 0,20 & 0,15 & 0,55 & 0,56 & 0,41 \\
\hline & Ind via NVP & 0,02 & $-0,02$ & $-0,14$ & 0,22 & 0,06 & 0,17 & 0,10 & 0,02 & 0,12 & $-0,11$ & $-0,26$ & $-0,15$ \\
\hline & Ind via MCG & $-0,20$ & $-0,32$ & $-0,31$ & $-0,30$ & $-0,32$ & $-0,23$ & $-0,38$ & $-0,34$ & $-0,28$ & $-0,48$ & $-0,48$ & $-0,34$ \\
\hline & $\mathrm{r}$ & $-0,06$ & $-0,14$ & $-0,21$ & 0,10 & 0,01 & 0,06 & $-0,03$ & $-0,12$ & $-0,01$ & $-0,04$ & $-0,17$ & $-0,08$ \\
\hline \multirow[t]{8}{*}{ MCG } & Dir sobre PROD & 0,41 & 0,51 & 0,61 & 0,52 & 0,57 & 0,45 & 0,59 & 0,63 & 0,53 & 0,74 & 0,75 & 0,59 \\
\hline & Ind via NVP & $-0,07$ & $-0,10$ & $-0,13$ & $-0,02$ & $-0,02$ & 0,01 & $-0,04$ & $-0,04$ & $-0,12$ & 0,00 & $-0,01$ & 0,03 \\
\hline & Ind via NSV & $-0,05$ & $-0,13$ & $-0,12$ & $-0,10$ & $-0,15$ & $-0,06$ & $-0,16$ & $-0,11$ & $-0,08$ & $-0,36$ & $-0,36$ & $-0,23$ \\
\hline & r & 0,29 & 0,29 & 0,35 & 0,39 & 0,40 & 0,40 & 0,39 & 0,48 & 0,33 & 0,38 & 0,38 & 0,38 \\
\hline & Coef. determinação & 0,77 & 0,74 & 0,77 & 0,87 & 0,88 & 0,85 & 0,88 & 0,89 & 0,80 & 0,86 & 0,85 & 0,85 \\
\hline & Variável residual & 0,48 & 0,51 & 0,48 & 0,35 & 0,35 & 0,38 & 0,35 & 0,33 & 0,45 & 0,37 & 0,39 & 0,39 \\
\hline & Número de condição & 16 & 16 & 20 & 31 & 33 & 25 & 34 & 39 & 18 & 40 & 41 & 32 \\
\hline & & 25 & 26 & 27 & 28 & 29 & 30 & 31 & 32 & 33 & 34 & 35 & 36 \\
\hline \multirow[t]{4}{*}{ NVP } & Dir sobre PROD & 0,86 & 0,93 & 0,82 & 0,79 & 0,78 & 0,83 & 0,82 & 0,82 & 0,82 & 0,80 & 0,77 & 0,88 \\
\hline & Ind via NSV & $-0,13$ & $-0,25$ & $-0,10$ & $-0,03$ & $-0,04$ & $-0,11$ & $-0,04$ & $-0,09$ & $-0,16$ & $-0,06$ & $-0,02$ & $-0,27$ \\
\hline & Ind via MCG & 0,00 & $-0,02$ & $-0,07$ & $-0,02$ & 0,07 & 0,07 & 0,07 & 0,07 & 0,08 & 0,02 & 0,07 & 0,11 \\
\hline & $\mathrm{r}$ & 0,73 & 0,67 & 0,65 & 0,75 & 0,81 & 0,80 & 0,85 & 0,80 & 0,74 & 0,76 & 0,82 & 0,72 \\
\hline \multirow[t]{4}{*}{ NSV } & Dir sobre PROD & 0,56 & 0,61 & 0,36 & 0,42 & 0,34 & 0,40 & 0,26 & 0,38 & 0,42 & 0,24 & 0,26 & 0,60 \\
\hline & Ind via NVP & $-0,20$ & $-0,38$ & $-0,22$ & $-0,06$ & $-0,08$ & $-0,22$ & $-0,13$ & $-0,19$ & $-0,30$ & $-0,19$ & $-0,06$ & $-0,39$ \\
\hline & Ind via MCG & $-0,54$ & $-0,51$ & $-0,34$ & $-0,43$ & $-0,34$ & $-0,36$ & $-0,26$ & $-0,41$ & $-0,40$ & $-0,26$ & $-0,31$ & $-0,45$ \\
\hline & $\mathrm{r}$ & $-0,17$ & $-0,28$ & $-0,20$ & $-0,07$ & $-0,08$ & $-0,19$ & $-0,13$ & $-0,21$ & $-0,28$ & $-0,21$ & $-0,11$ & $-0,25$ \\
\hline \multirow[t]{7}{*}{ MCG } & Dir sobre PROD & 0,77 & 0,86 & 0,62 & 0,71 & 0,56 & 0,60 & 0,47 & 0,61 & 0,71 & 0,49 & 0,56 & 0,73 \\
\hline & Ind via NVP & 0,00 & $-0,02$ & $-0,10$ & $-0,02$ & 0,09 & 0,09 & 0,13 & 0,09 & 0,09 & 0,03 & 0,09 & 0,14 \\
\hline & Ind via NSV & $-0,39$ & $-0,36$ & $-0,20$ & $-0,25$ & $-0,21$ & $-0,24$ & $-0,15$ & $-0,25$ & $-0,24$ & $-0,12$ & $-0,14$ & $-0,37$ \\
\hline & $\mathrm{r}$ & 0,38 & 0,47 & 0,33 & 0,44 & 0,45 & 0,45 & 0,45 & 0,45 & 0,56 & 0,39 & 0,51 & 0,49 \\
\hline & Coef. determinação & 0,82 & 0,86 & 0,66 & 0,87 & 0,86 & 0,86 & 0,87 & 0,85 & 0,89 & 0,75 & 0,89 & 0,84 \\
\hline & Variável residual & 0,43 & 0,38 & 0,58 & 0,36 & 0,37 & 0,38 & 0,36 & 0,38 & 0,34 & 0,50 & 0,33 & 0,40 \\
\hline & Número de condição & 35 & 51 & 16 & 38 & 33 & 37 & 35 & 37 & 52 & 18 & 41 & 44 \\
\hline
\end{tabular}




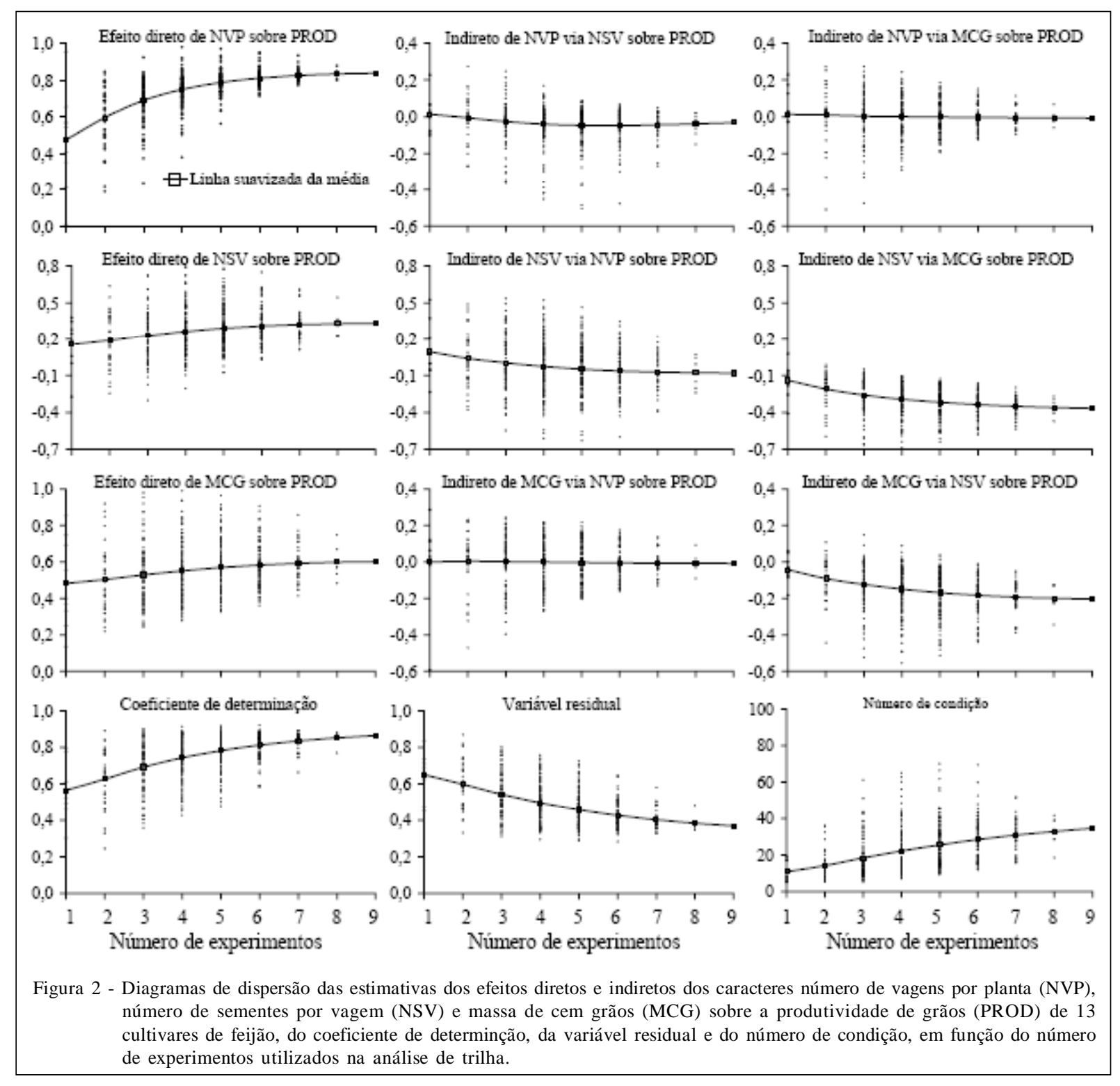

de caracteres, confirmou diferença significativa entre os coeficientes. Então, a diferença entre os experimentos, quanto aos coeficientes de correlação, leva a distintos resultados da análise de trilha, quando ela for realizada com apenas um experimento. Portanto, o número de experimentos necessários para a análise de trilha merece ser investigado, a fim de obter resultados com confiabilidade.

De maneira geral, em sete experimentos $(1,2$, 3, 4, 5, 6 e 9), os coeficientes de correlação linear de Pearson ( $\mathrm{r}$ ) entre a variável explicativa número de vagens por planta (NVP) e a variável principal produtividade de grãos (PROD) foram positivos $(0,4175 \leq \mathrm{r} \leq 0,8279)$ e de sinal e magnitude semelhante ao efeito direto sobre $\operatorname{PROD}(0,3526 \leq$ efeito direto $\leq 0,9539)$, o que revela relação de causa e efeito do NVP sobre a PROD. No entanto, em dois experimentos ( 8 e 9), os baixos valores de $r(\leq 0,1492)$ e dos efeitos diretos $(\leq 0,2132)$ explicam a ausência de relação de causa e efeito do NVP sobre a PROD (Tabela 1). Portanto, esses resultados comprovam que análises de trilha a partir de dados de experimentos individuais levam a resultados distintos. Raciocínio semelhante pode ser aplicado para visualizar os distintos resultados das análises de trilha das variáveis explicativas número de sementes por vagem (NSV) e massa de cem grãos (MCG) sobre a variável principal produtividade de grãos (PROD) a partir dos dados de experimentos individuais.

As análises de correlação e de trilha, realizadas com os dados dos nove experimentos, 


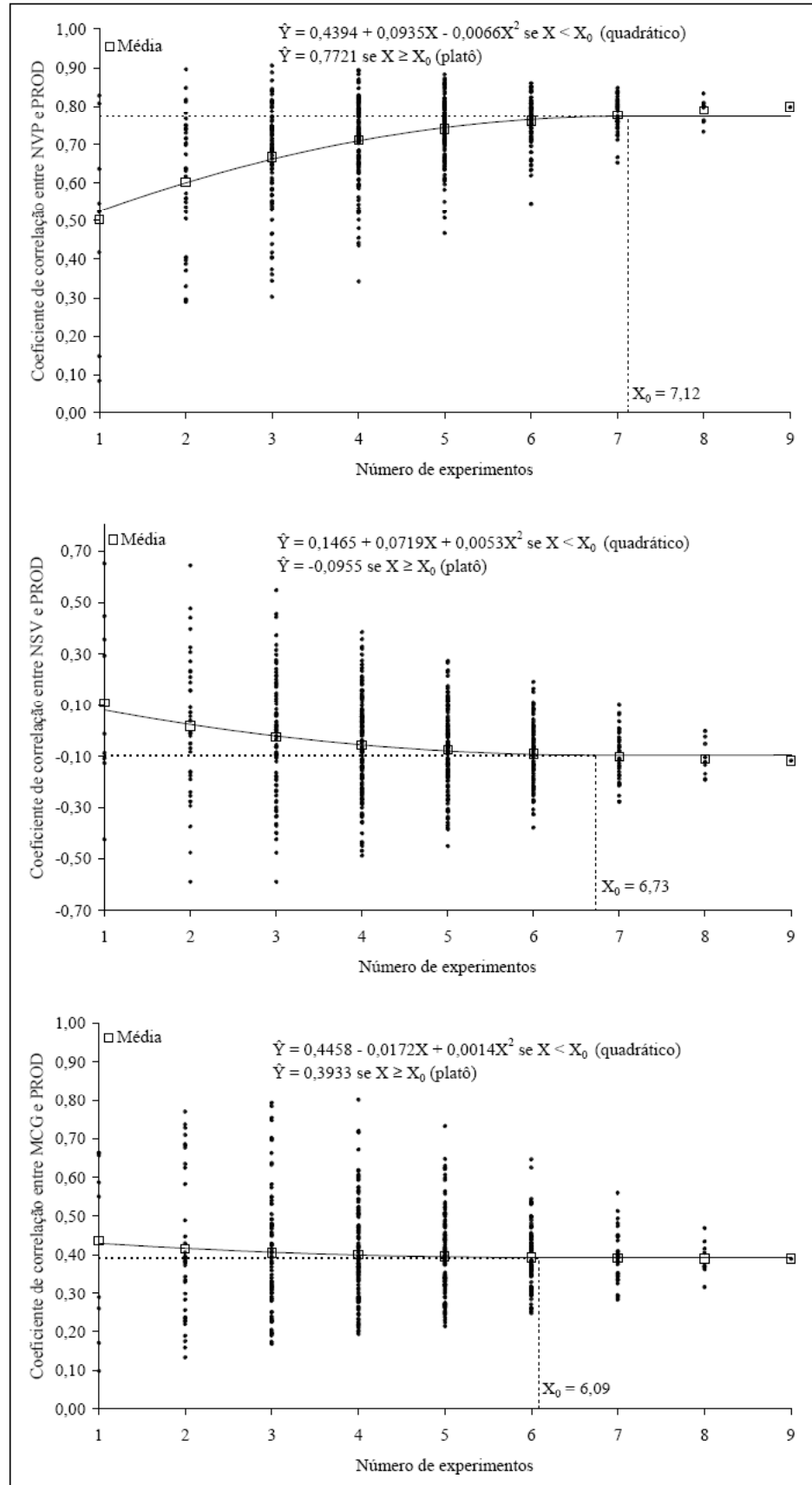

Figura 3 - Equações estimadas do modelo quadrático de resposta com platô de $\mathrm{Y}$ (coeficientes de correlação linear de Pearson entre os caracteres número de vagens por planta (NVP), número de sementes por vagem (NSV) e massa de cem grãos (MCG) versus produtividade de grãos (PROD) de 13 cultivares de feijão, avaliadas em nove experimentos) em função da variável $\mathrm{X}$ (número de experimentos) e número de experimentos para a análise de trilha $\left(\mathrm{X}_{0}\right)$. se inferir que há associação linear de causa e efeito entre esses caracteres. Por outro lado, não ocorreram associações lineares significativas entre NSV e PROD ( $\mathrm{r}=-0,1162)$ e entre MCG e PROD ( $\mathrm{r}=3894)$, e os efeitos diretos do $\operatorname{NSV}(0,3316)$ e da MCG $(0,6611)$ sobre a PROD foram, comparativamente, inferiores ao efeito direto do NVP sobre a PROD (0,8373) (Tabela 1). Esses resultados evidenciam a não existência de associação linear de causa e efeito entre o NSV e a PROD e entre a MCG e a PROD. Então, de maneira geral, pode-se inferir que as plantas com maior número de vagens estão associadas a plantas com maior produtividade de grãos e, portanto, o caráter número de vagens por planta, pode ser utilizado para seleção indireta de plantas. Salienta-se que esses resultados devem ser vistos com ressalvas e poderiam ser diferentes no caso de serem oriundos de matrizes de correlação genotípica. Resultados semelhantes foram encontrados por DUARTE \&ADAMS (1972), COIMBRA et al. (1999), KUREK et al. (2001), FURTADO et al. (2002) e DURSUN (2007).

Os efeitos diretos e indiretos, estimados por meio da análise de trilha, dependem dos coeficientes de correlação linear de Pearson (r). Portanto, é importante investigar o número de experimentos a partir do qual o valor de r estabiliza e, como consequência, a inclusão de dados de mais experimentos não altera os resultados da análise de trilha. Por meio do modelo quadrático de resposta com platô das variáveis $\mathrm{Y}$ (coeficientes de correlação linear de Pearson entre NVP e PROD, NSV e PROD e MCG e PROD) em função da variável X (número de experimentos), foi constatado que, a partir de 7,12, 6,73 e 6,09 experimentos, o valor de r, respectivamente, entre NVP e PROD, revelaram que houve associação linear positiva e significativa entre NVP e PROD ( $r=0,7989)$ e o efeito direto foi de mesmo sinal e magnitude semelhante $(0,8373)$. Além disso, os efeitos indiretos via NSV $(-0,0318)$ e via MCG $(-0,0066)$ foram desprezíveis. Portanto, pode-
NSV e PROD e MCG e PROD, estabilizou (Tabela 2 e Figura 3). Assim, a média, arredondada para cima, dos três valores de $\mathrm{X}_{0}$ (sete experimentos) foi considerada o número necessário de experimentos para a análise de trilha (Tabela 2).

Ciência Rural, v.41, n.4, abr, 2011. 
De maneira geral, a variabilidade das estimativas dos coeficientes de correlação linear de Pearson e dos respectivos efeitos diretos e indiretos dos caracteres estudados, bem como do coeficiente de determinação, da variável residual e do número de condição, diminui com o acréscimo do número de experimentos (Figuras $2 \mathrm{e} 3$ ). Esses resultados revelam aumento da confiabilidade dos resultados com o acréscimo do número de experimentos. Revelam, também, de maneira geral, que os resultados da análise de trilha a partir de sete, oito ou nove experimentos são similares. Assim, caso a finalidade principal deste estudo fosse, apenas, fazer a análise de trilha, o uso da análise conjunta dos nove experimentos seria um procedimento adequado. No entanto, este estudo visou a determinar o número de experimentos necessários para a análise de trilha em feijão e, portanto, as análises individuais e as combinações dos nove experimentos tomados dois a dois, três a três, ..., e oito a oito experimentos, além da análise conjunta dos nove experimentos, foram particularmente importantes, por proporcionar uma quantidade maior de cenários.

$\mathrm{Na}$ prática, principalmente por parte dos melhoristas de plantas, os resultados deste estudo devem ser vistos com extrema cautela e considerados como sendo de um caso particular, isto é, os resultados deste estudo foram obtidos a partir da matriz de correlação fenotípica de quatro caracteres de treze cultivares, avaliadas em nove experimentos conduzidos em duas épocas de cultivo em um mesmo local. Portanto, não necessariamente o pesquisador deve conduzir sete experimentos, para, somente após isso, realizar a análise de trilha. É importante e necessário considerar diversos aspectos que ainda devem ser investigados. Provavelmente, o número necessário de experimentos para análise de trilha, com base em dados de experimentos conduzidos somente nas épocas de safra ou somente na safrinha, seja inferior a sete experimentos, pelo fato de os ambientes, comumente, serem mais homogêneos do que entre as épocas de cultivo. Particularmente, neste estudo, a separação dos nove ambientes em safra (cinco ambientes) e safrinha (quatro ambientes) reduziria o número de simulações de 511 para $31 \mathrm{com}$ os experimentos de safra e $17 \mathrm{com}$ os experimentos de safrinha. Com isso, o uso do modelo quadrático de resposta com platô para determinar o número de experimentos deveria ser repensado devido ao reduzido número de experimentos simulados. Além desses aspectos, o número de experimentos a partir de matrizes de coeficientes de correlação genotípica e residual pode ser diferente do obtido neste trabalho.
Diferentes cenários formados pela combinação de distintos números de indivíduos (cultivares), números de variáveis, número de repetições, número de experimentos, experimentos agrupados por época de cultivo (safra e safrinha), distintas matrizes de coeficientes de correlação (fenotípica, genotípica e residual) poderiam ser simulados e estudados antes dessas informações serem generalizadas. É provável que o número de experimentos possa ser diminuído com o uso de mais repetições, como, por exemplo, quatro repetições, conforme recomendam CARGNELUTTI FILHO et al. (2009b), para ensaios de avaliação de cultivares de feijão, mantendo a mesma precisão. Enfim, do ponto de vista prático, embora a execução de mais experimentos possa refletir em demora na divulgação de resultados, é importante que o pesquisador, ao fazer um estudo de relações lineares entre caracteres, tenha um determinado número de observações que deem confiabilidade em suas conclusões. Também é importante que a metodologia apresentada neste trabalho seja testada em outros cenários e aperfeiçoada, se necessário. Enfim, resultados distintos entre análises de trilha podem estar relacionados a uma amostra (número de experimentos) insuficiente.

\section{CONCLUSÃO}

Na região da depressão central do Estado do Rio Grande do Sul, sete experimentos são suficientes para a análise de trilha do número de vagens por planta, do número de sementes por vagem e da massa de cem grãos sobre a produtividade de grãos de cultivares de feijão (Phaseolus vulgaris L.).

\section{AGRADECIMENTOS}

Ao Conselho Nacional de Desenvolvimento Científico e Tecnológico (CNPq) e à Coordenação de Aperfeiçoamento de Pessoal de Nível Superior (CAPES), pela concessão de bolsas aos autores.

\section{REFERÊNCIAS}

CARGNELUTTI FILHO, A. et al. Número necessário de experimentos para a análise de agrupamento de cultivares de feijão. Ciência Rural, v.39, p.371-378, 2009a. Disponível em: <http:/ /www.scielo.br/scielo.php? script $=$ sci_arttext\&pid=S0103$84782009000200009 \& \operatorname{lng}=$ pt\&nrm=iso $>$. Acesso em: 22 jul. 2010. doi: 10.1590/S0103-84782008005000059.

CARGNELUTTI FILHO, A. et al. Número necessário de experimentos para a comparação de cultivares de feijão. Ciência Rural, v.36, p.1701-1709, 2006. Disponível em: <http:// www.scielo.br/scielo.php?script $=$ sci_arttext\&pid $=$ S0103- 
$84782006000600006 \& \operatorname{lng}=$ pt\&nrm=iso $>$. Acesso em: 22 jul. 2010. doi: $10.1590 /$ S0103-84782006000600006.

CARGNELUTTI FILHO, A. et al. Número de repetições para a comparação de cultivares de feijão. Ciência Rural, v.39, p.2419-2424, 2009b. Disponível em: <http://www.scielo.br/ scielo.php? script =sci_art text \& pid=S0103$84782009000900006 \& \operatorname{lng}=$ pt\&nrm=iso $>$. Acesso em: 22 jul. 2010. doi: 10.1590/S0103-84782009000900006.

COIMBRA, J.L.M. et al. Análise de trilha I: análise do rendimento de grãos e seus componentes. Ciência Rural, v.29, p.213-218, 1999. Disponível em: <http://www.scielo.br/ scielo.php? script $=$ sci_arttext\&pid=S0103$84781999000200005 \& \operatorname{lng}=$ pt\&nrm=iso $>$. Acesso em: 22 jul. 2010. doi: 10.1590/S0103-84781999000200005.

CRUZ, C.D. Programa genes: estatística experimental e matrizes. Viçosa: UFV, 2006. 285p.

CRUZ, C.D.; CARNEIRO, P.C.S. Modelos biométricos aplicados ao melhoramento genético. Viçosa: UFV, 2003. V.2, 585p.

CRUZ, C.D.; REGAZZI, A.J. Modelos biométricos aplicados ao melhoramento genético. 2.ed. Viçosa: UFV, 1997. 390p.

DUARTE, R.A.; ADAMS, M.W. A path coefficient analysis of some yield component interrelation in field beans (Phaseolus vulgaris L). Crop Science, v.12. p.579-582, 1972.
DURSUN, A. Variability, heritability and correlation studies in bean (Phaseolus vulgaris L.) genotypes. Word Journal of Agricultural Sciences, v.3, p.12-16, 2007.

FERREIRA, D.F. Estatística básica. 2.ed. Lavras: UFLA, 2009. 664p.

FURTADO, M.R. et al. Análise de trilha do rendimento do feijoeiro e seus componentes primários em monocultivo e em consórcio com a cultura do milho. Ciência Rural, v.32, p.217-220, 2002. Disponível em: <http://www.scielo.br/ scielo.php? script =sci_art text \& pid=S 0103 $84782002000200006 \& \operatorname{lng}=$ pt\&nrm=iso $>$. Acesso em: 22 jul. 2010. doi: $10.1590 / \mathrm{S} 0103-84782002000200006$.

HAIR, J.F. et al. Análise multivariada de dados. 5.ed. Porto Alegre: Bookman, 2005. 593p.

KUREK, A.J. et al. Análise de trilha como critério de seleção indireta para rendimento de grãos em feijão. Revista Brasileira de Agrociência, v.7, p.29-32, 2001.

MONTGOMERY, D.C.; PECK, E.A. Introduction to linear regression analysis. New York: John Wiley \& Sons, 1982. $504 \mathrm{p}$.

REZENDE, D.M.L.C. et al. Ajuste de modelos de platô de resposta para a exigência de zinco em frangos de corte. Ciência e Agrotecnologia, v.31, p.468-478, 2007. Disponível em: <http:/ $/ \mathrm{www}$. scielo.br/scielo.php? script=sci_arttext\&pid=S1413$70542007000200030 \& \operatorname{lng}=$ pt\&nrm $=$ iso $>$. Acesso em: 22 jul. 2010. doi: $10.1590 / \mathrm{S} 1413-70542007000200030$. 